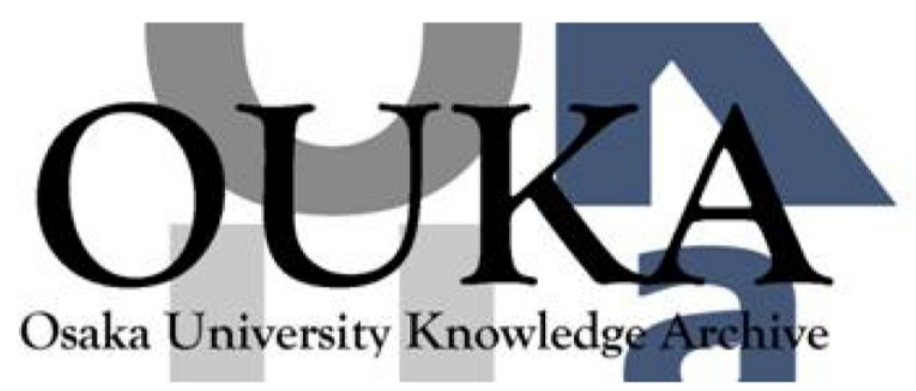

\begin{tabular}{|c|l|}
\hline Title & Raman scattering gain in D00-PPV films \\
\hline Author(s) & $\begin{array}{l}\text { Shkunov, Maxim N. ; Gellermann, Werner; Fuji i, } \\
\text { Akihiko et al. }\end{array}$ \\
\hline Citation & $\begin{array}{l}\text { Proceedings of SPIE - The International Society } \\
\text { for Optical Eng ineering. 3145 p. 88-p. 94 }\end{array}$ \\
\hline Issue Date & $1997-12-01$ \\
\hline oaire:version & VoR \\
\hline URL & https://hdl. handle. net/11094/76943 \\
\hline rights & \\
\hline Note & \\
\hline
\end{tabular}

Osaka University Knowledge Archive : OUKA

https://ir. Library. osaka-u. ac. jp/

Osaka University 


\title{
Raman scattering gain in DOO-PPV films
}

\author{
M.N. Shkunov", W. Gellermann", A.Fujii, ${ }^{\mathrm{a}, \mathrm{b}}, \mathrm{K}$. Yoshino ${ }^{\mathrm{b}}$ and Z.V.Vardeny"
}

'Dept. of Physics, University of Utah, Salt Lake City, Utah 84112

'Dept. of Electronic Engineering, Faculty of Engineering, Osaka University, 2-1 Yamada-Oka, Suita, Osaka 565, Japan

\begin{abstract}
We have investigated the optical emission characteristics of poly(2,5-dioctyloxy-p-phenylenevinylene) (DOOPPV) thin films under high intensity ps pulsed laser excitation ( $I_{\text {pump }}>1 \mathrm{MW} / \mathrm{cm}^{2}$ ). We observed that the emission spectrum consist of a spectrally narrowed emission (SNE) band (FWHM $\sim 10 \mathrm{~nm}$ ) and superimposed sharp spectral lines. High resolution spectral measurements conducted at room and liquid nitrogen temperatures show that the spectral width of these lines is less than $1 \mathrm{~nm}$. The lines peak positions are independent of film thickness, however, they shifts upon changing the excitation wavelength. Also, the excitation intensity dependence of these narrow lines is found to be different from that of the SNE band. We argue that the narrow lines are due to Raman scattering gain of the $1^{\text {sa }}, 2^{\text {nd }}$ and $3^{\mathrm{d}}$ order Raman active modes of DOO-PPV.
\end{abstract}

Keywords: DOO-PPV, Raman scattering gain, stimulated emission, strongly coupled vibrations.

\section{INTRODUCTION}

The investigation and explanation of the exciton emission behavior in luminescent conducting polymer thin films under high excitation intensities have recently drawn considerable attention ${ }^{1-7}$. The high optical gain achieved in these materials results in dramatic emission spectral narrowing and radiative lifetime shortening when the excitation intensity exceeds a certain threshold value. For example, for films of the soluble derivative of PPV, namely DOO-PPV, the spectral narrowing process occurs as bright emission with about ten-fold reduced spectral width and subpicosecond time duration, when the exciton density exceeds a threshold level of $\sim 5 \times 10^{17} \mathrm{~cm}^{-3}$. This nonlinear emission behavior has been attributed to several processes such as amplified spontaneous emission ${ }^{3}$, cooperative emission or superfluorescence ${ }^{4,5}$ and mirrorless lasing ${ }^{6}$. We will refer to this emission simply as spectrally narrowed emission (SNE) throughout this paper.

In this work we report novel spectral features observed in the emission of DOO-PPV films which occur in addition to, or on top of the SNE band.

\section{EXPERIMENTAL}

For our measurements we used thin DOO-PPV films spin-coated on glass substrates. The samples were excited at three different wavelengths : 532,558 and $563.5 \mathrm{~nm}$, respectively; the laser pulse duration was $\sim 100 \mathrm{ps}$ and the pulse energies varied from 0.1 to $50 \mu \mathrm{J}$. The excitation light was focused onto the polymer film within an area of $\sim 1 \mathrm{~mm}^{2}$. The emission spectra were measured with a SPEX $0.6 \mathrm{~m}$ triple spectrometer, employing 300, 600 and 1200 grooves/mm gratings and a cooled CCD array. To avoid oxidation, the samples were kept in a cryostat under vacuum of $10^{-3}$ torr, where the temperature of the samples could be changed between 80 and $300 \mathrm{~K}$. 


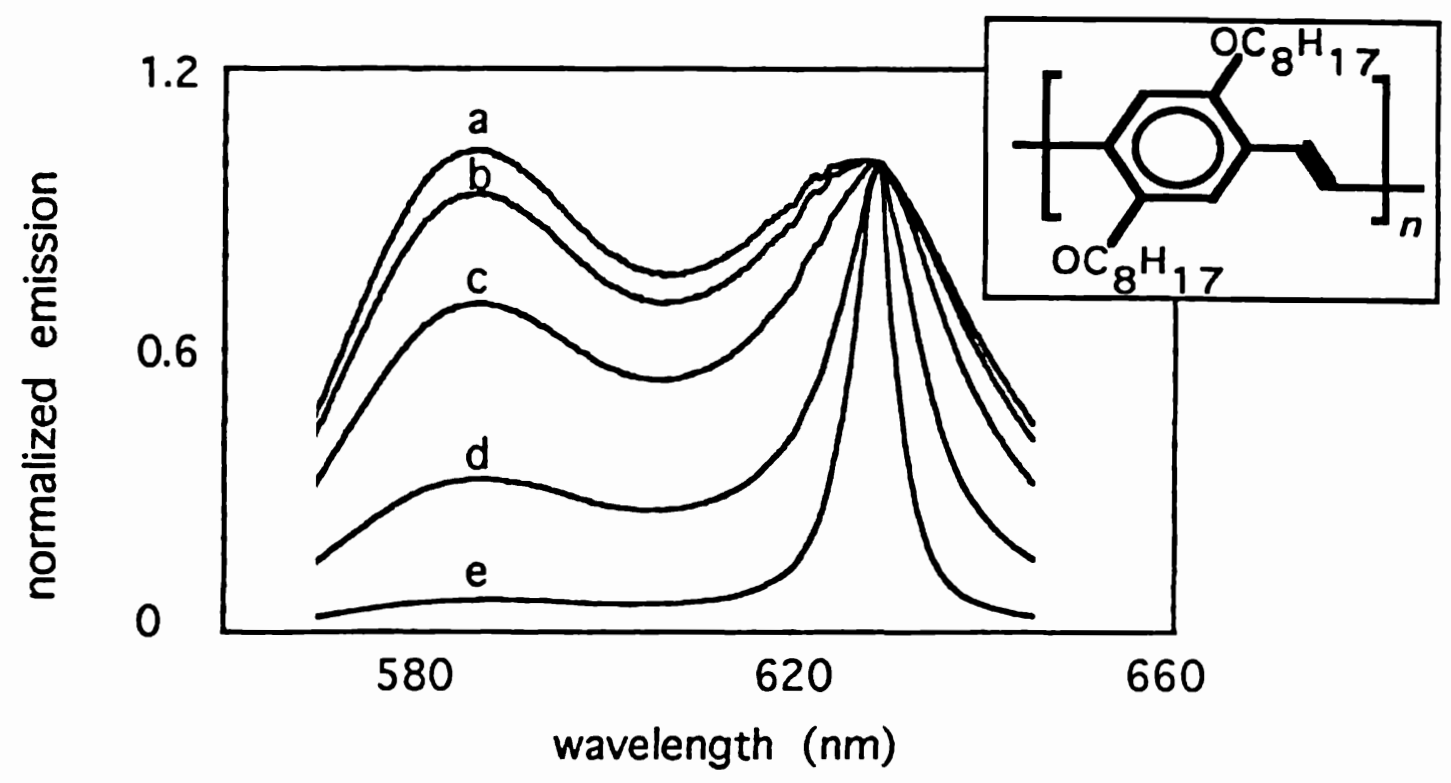

Figure 1. Normalized emission spectra of DOO-PPV film at room temperature obtained with $532 \mathrm{~nm}$ excitation, pulse width $\sim 100 \mathrm{ps,}$, repetition rate $100 \mathrm{~Hz}$. The excitation energies per pulse are: (a) $0.11 \mu \mathrm{J}$, (b) $0.12 \mu \mathrm{J}$, (c) $0.15 \mu \mathrm{J}$, (d) $0.20 \mu \mathrm{J}$, (e) $0.30 \mu$ J. The DOO-PPV chemical structure is shown in the insert.

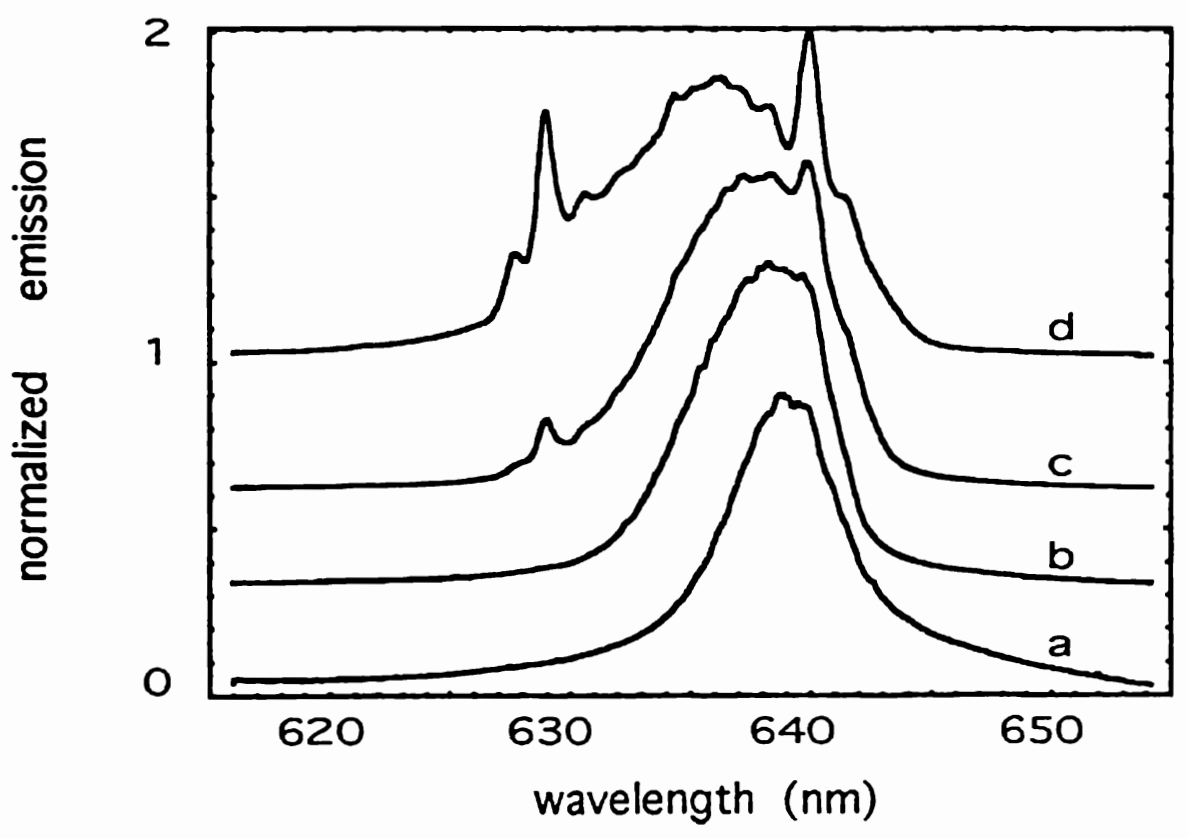

Figure 2. Emission spectra for higher excitation intensities, at $80 \mathrm{~K}$. Pump energies are: (a) $0.1 \mu \mathrm{J}$, (b) $0.65 \mu \mathrm{J}$, (c) $1.6 \mu \mathrm{J}$ and (d) $5.3 \mu \mathrm{J}$. 


\section{RESULTS AND DISCUSSION}

For $532 \mathrm{~nm}$ excitation at room temperature the emission spectrum of DOO-PPV follows the typical spectral narrowing process in conducting polymer films: when the excitation energy is increased from 0.1 to $0.3 \mu \mathrm{J} / \mathrm{pulse}$ the spectral width of the polymer emission collapses from a FWHM of $\sim 80 \mathrm{~nm}$ to $7 \mathrm{~nm}$ (Fig.1). However, a further increase of the pump energy leads to the appearance of new, sharp spectral features superimposed on the SNE band. This is illustrated in Fig.2 where we show the emission of DOO-PPV films measured at $80 \mathrm{~K}$. As the pump energy is further increased from 0.1 to $5.3 \mu \mathrm{J} / \mathrm{pulse}$, two sharp peaks emerge on the shoulders of the SNE band at 629 and $640 \mathrm{~nm}$, respectively. The FWHM of these sharp lines is less than $1 \mathrm{~nm}$, and thus is significantly narrower than the SNE band (Fig.2).

Two effects are conceivable for the origin of the observed sharp lines: (1) the presence of a microcavity mode structure or (2) the presence of a nonlinear process associated with Raman scattering, namely Raman gain.

Considering the first possibility, a cavity length estimate of a possible microcavity, formed by the polymer-air and polymer-glass interfaces, gives $d=10 \mu$ when using the relation ${ }^{8}$

$$
d=\mathrm{c} / 2 \mathrm{n} \Delta \mathrm{v},
$$

where $d$ - cavity length, $\mathrm{c}$ - speed of light, $\mathrm{n}$ - refraction index of the medium, $\Delta \mathrm{v}$ - difference in mode frequency. This value for $d$ is at least one order of magnitude larger than the polymer film thickness used in our measurements. Another consequence of a microcavity consideration is that we would expect the mode spacing to be different for various film thicknesses. In Fig. 3 we show DOO-PPV emission spectra for two films with different thicknesses: $240 \mathrm{~nm}$ and $900 \mathrm{~nm}$ thick, respectively. In both cases the spectral separation of the narrow lines is the same.

From these two observations we conclude that microcavity effects can be ruled out as giving rise to the narrow emission lines.

The excitation intensity needed for observation of the narrow lines was in the order of $2+50 \mathrm{MW} / \mathrm{cm}^{2}$; these are quite high intensity levels, at which nonlinear optical phenomena are expected to play an important role. This fact, together with the sharpness of the narrow emission lines, points at the possibility that stimulated Raman scattering (SRS) is present in our measurements. Under this assumption we expect to observe the following features:

(i) the spectral position of the emission lines should change with the shift of the excitation wavelength.

(ii) the output intensity $I_{S R S}$ should vary exponentially with the pump intensity $I_{\text {pump }}$, according to the relation?

$$
\mathrm{I}_{\text {SRS }}=\mathrm{I}_{\text {SRS }}(0)^{*} \exp \left(\mathrm{gI}_{\mathrm{pump}} 0\right.
$$

where $g$ is the gain coefficient, and $\ell$ - the length of the active medium.

(iii) we expect to observe a threshold effect as well as gain saturation.

(iv) the Raman lines might be observed not only at first order but also at higher orders.

To perform a more detailed study related to the possibility of Raman gain, we measured the $1^{\text {tt }}$ order Raman scattering spectrum of DOO-PPV at low excitation intensities. To avoid the strong luminescence background, we used long-wavelength excitation with a CW Ti:sapphire laser operating at $752 \mathrm{~nm}$. The Raman spectrum is given in Fig.4 and shows several strong lines located at $1584,1313,1285,1117$ and $968 \mathrm{~cm}^{-1}$ respectively. According to Sakamoto et al. ${ }^{10}$, who measured the Raman spectrum of unsubstituted PPV, the strongest Raman line at $1584 \mathrm{~cm}^{-1}$ is assigned to the polymer ring mode corresponding to the $v_{84}$ of benzene in the Wilson notation. We thus expect this phonon line to strongly participate in the Raman gain process at high excitation intensities.

Figure 5 shows the emission spectra of a DOO-PPV film at high excitation intensity for two additional excitation wavelengths, namely 558 and $563.5 \mathrm{~nm}$. In both cases the SNE band is centered at $630 \mathrm{~nm}$ and remains unshifted. However, a very intense, single, sharp emission line appears on the high energy side of the SNE band. From high resolution measurements we determined the line width of this sharp line to be less than $3 \AA$. The position of this sharp line is shifted at $558 \mathrm{~nm}$ excitation compared to $563.5 \mathrm{~nm}$ excitation, and this frequency shift exactly corresponds to the froquency difference of the two excitation wavelengths. Therefore, we have a clear indication for the presence of a first order Raman scattering gain in our DOO-PPV films.

To identify the Raman active modes participating in the SNE at high excitation intensities we have plotted the first-order Raman spectrum of DOO-PPV together with the polymer emission for the $563.5 \mathrm{~nm}$ excitation (Figure 6). It 


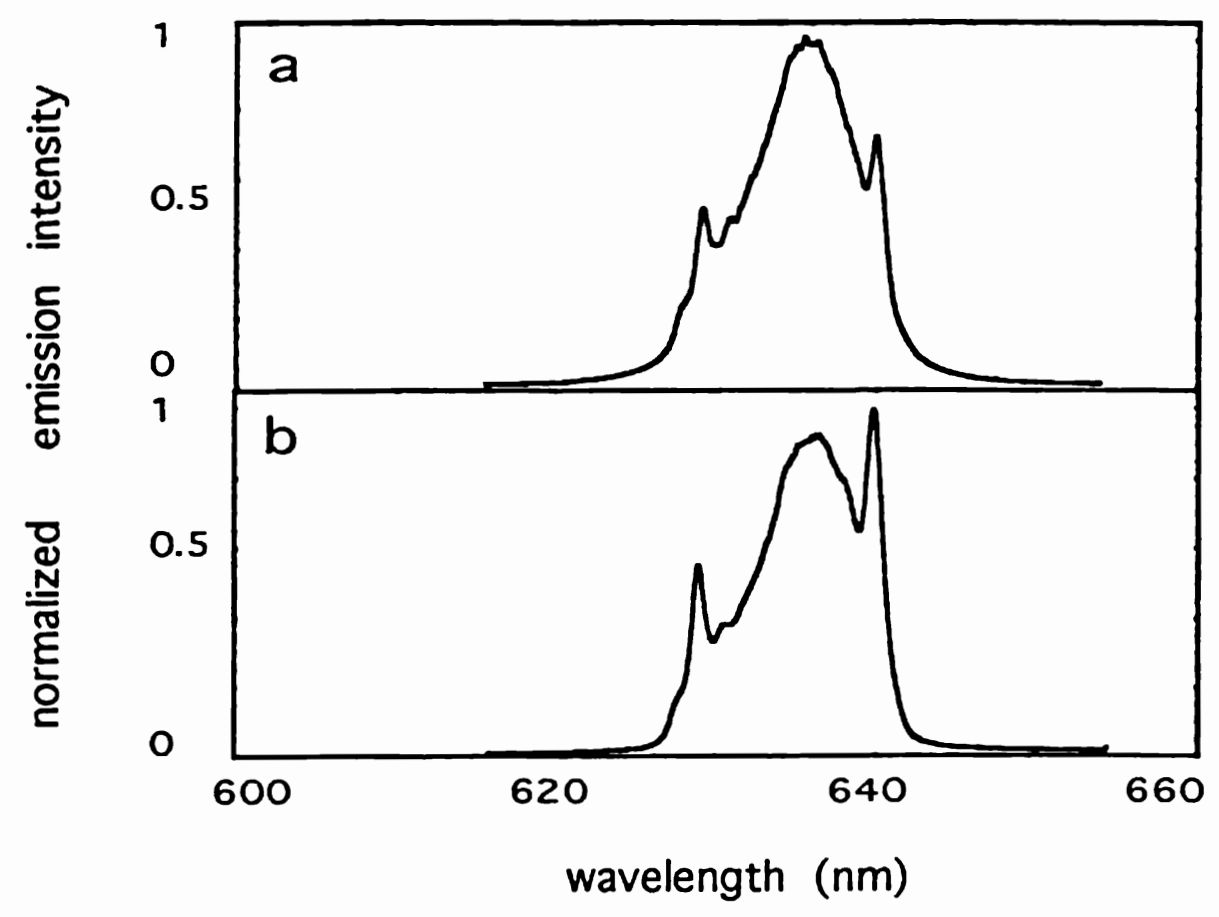

Figure 3. DOO-PPV emission spectra for two films of different thickness d, at $80 \mathrm{~K}$, where the excitation wavelength is $532 \mathrm{~nm}$. (a) d $\sim 240 \mathrm{~nm}$, (b) d 900 nm.

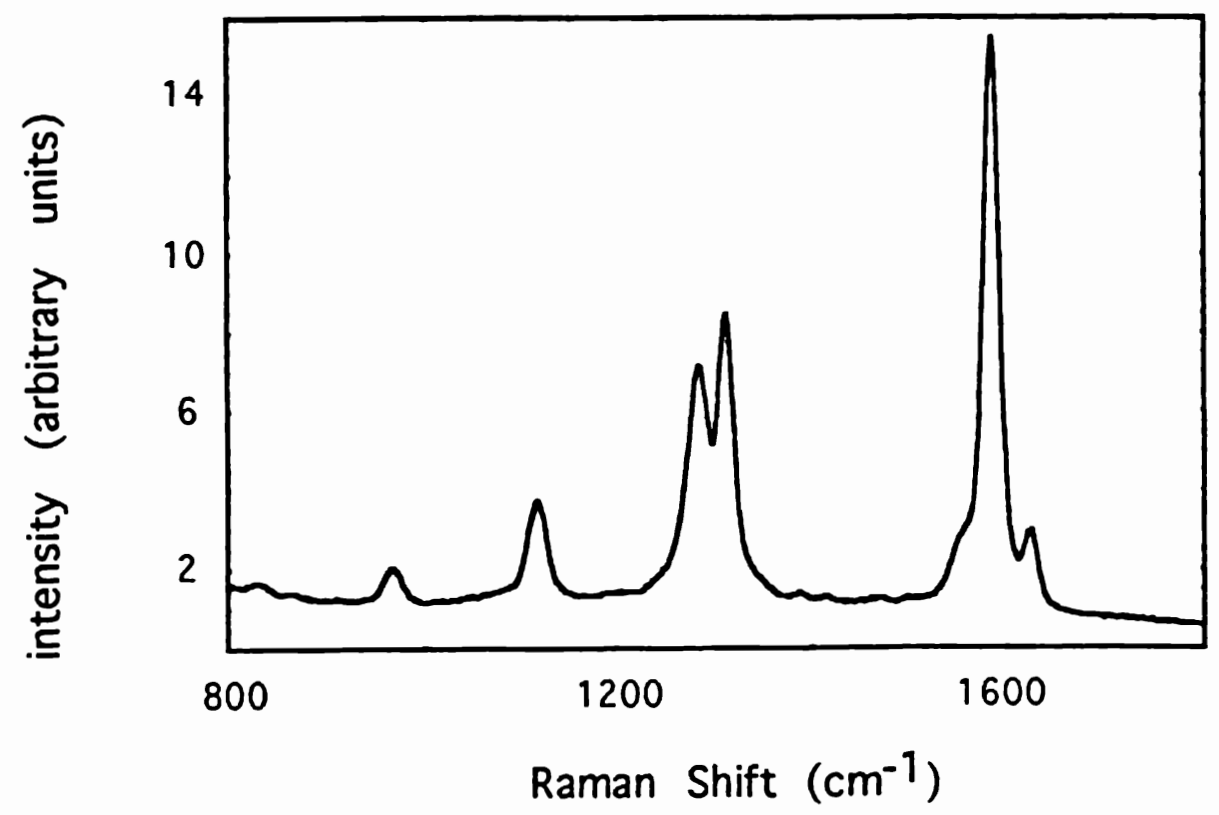

Figure 4. DOO-PPV Raman spectrum at low excitation intensity. Excitation source : $10 \mathrm{~mW}$ Ti:sapphire laser at $752 \mathrm{~nm}$. 


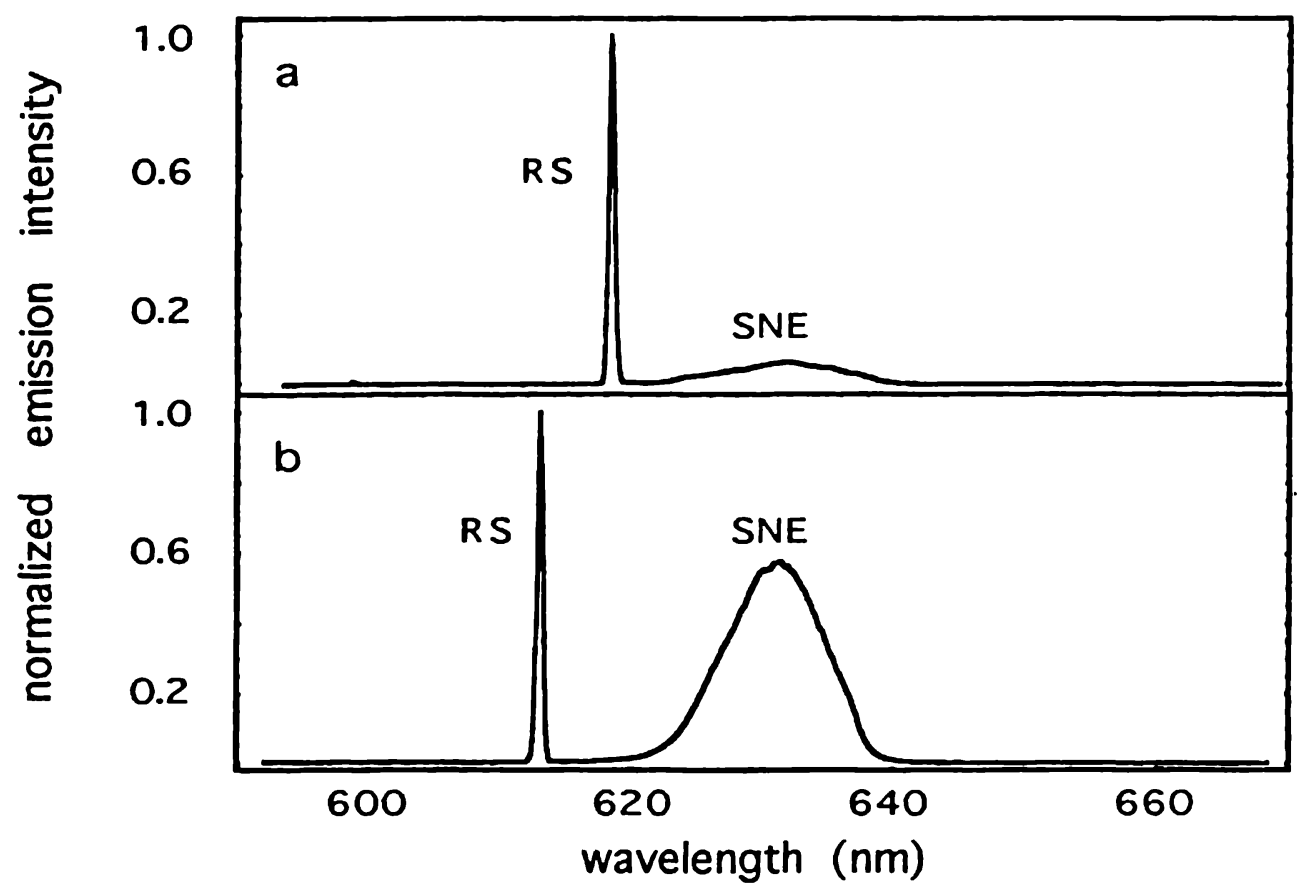

Figure 5. DOO-PPV emission spectra at high excitation intensities for two different excitation wavelengths: (a) $\lambda_{\text {exc }}=563.5 \mathrm{~nm}$, (b) $\lambda_{\text {exc }}=558 \mathrm{~nm}$. The amplified first order Raman scattering lines (RS) are shifted from spectrally narrowed emision (SNE) band.

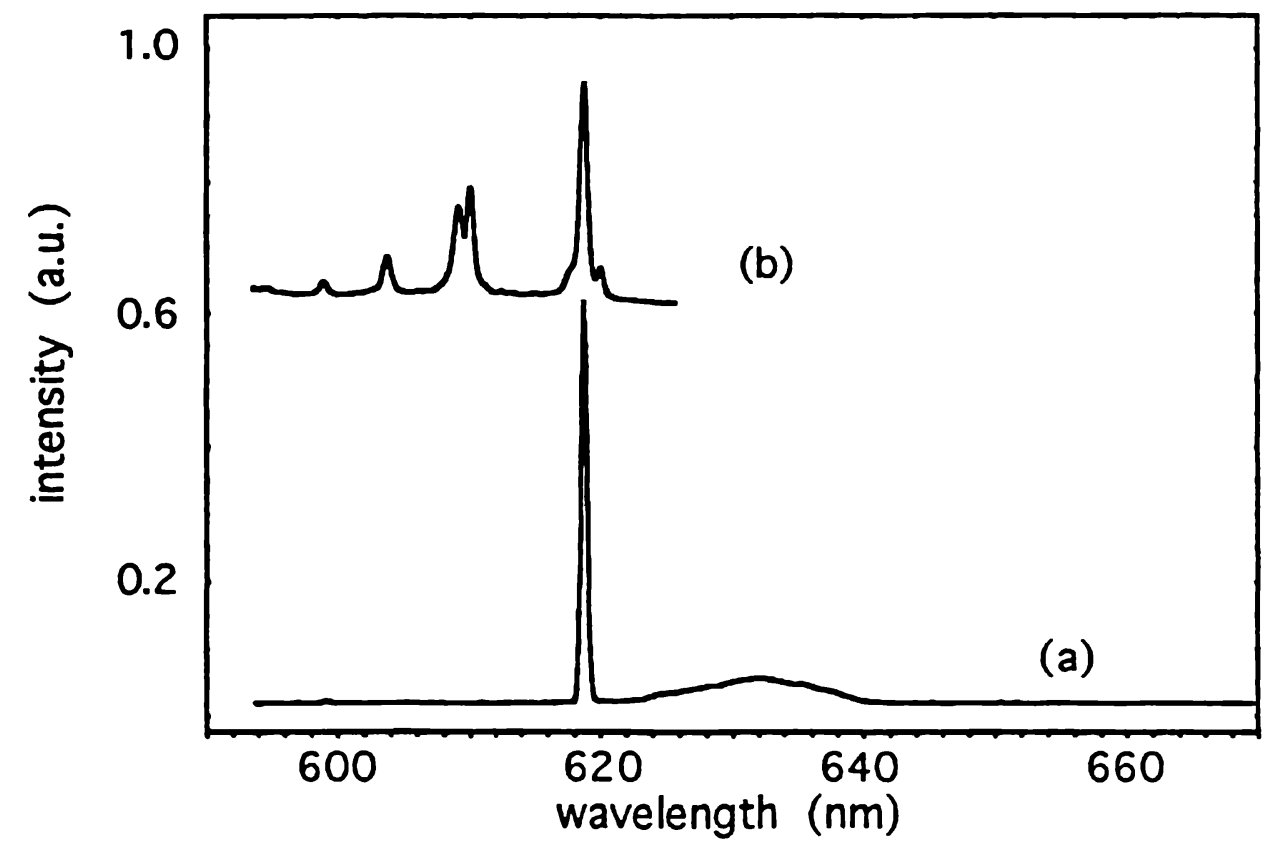

Figu re 6. DOO-PPV emission at high intensity (a) and first order Raman spectrum, obtained at low excitation intensity (b). Excitation wavelength is $563.5 \mathrm{~nm}$. 


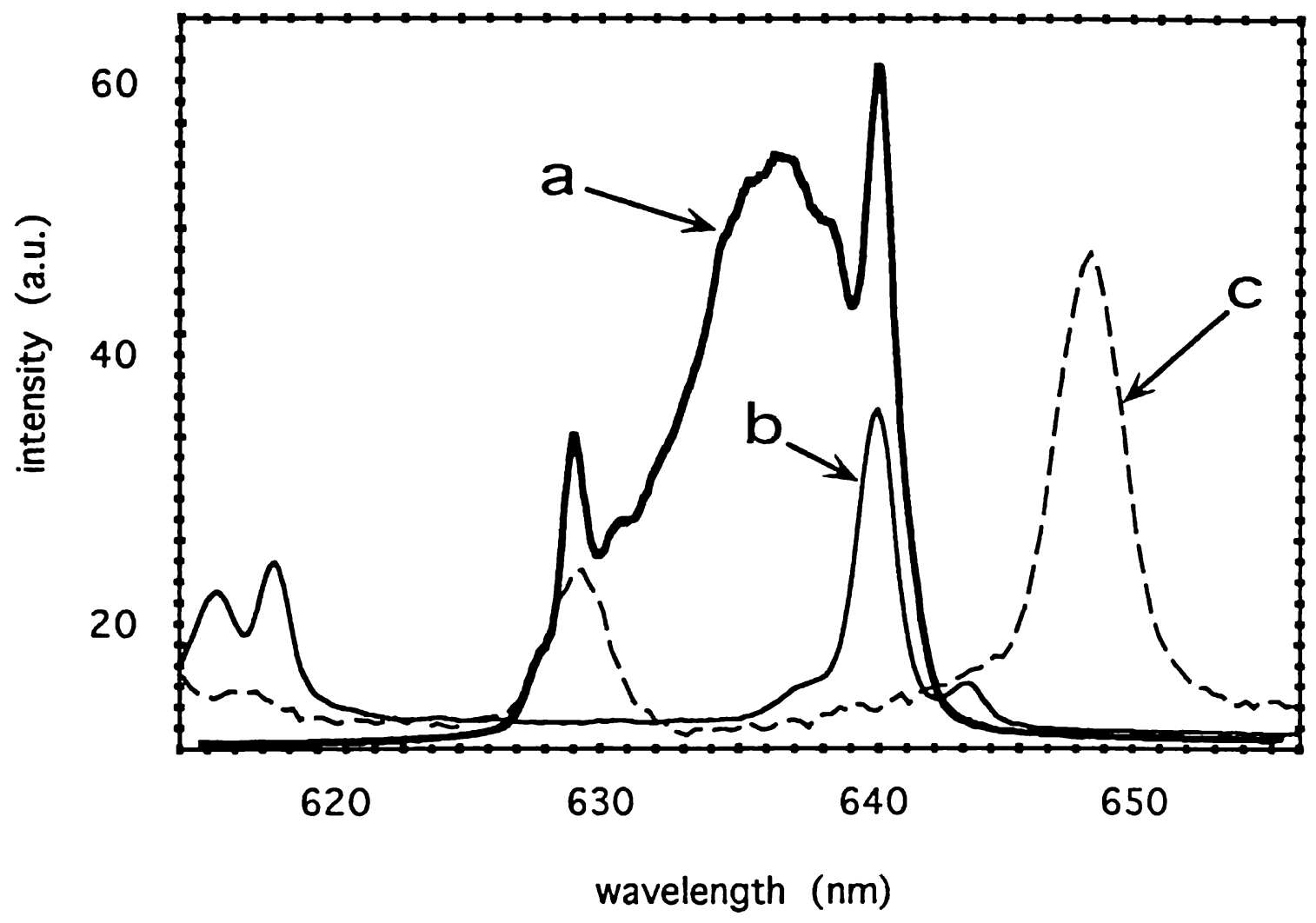

Figure 7. (a) DOO-PPV emission spectrum at high excitation intensity. Experimental conditions: $\lambda_{\text {exc }}=532 \mathrm{~nm}, \mathrm{E}_{\text {exc }}=7 \mu \mathrm{J}, \mathrm{T}=80 \mathrm{~K}$;

(b) Second order Raman spectrum of 1285,1313 and $1584 \mathrm{~cm}^{-1}$ modes is shown as a thin line.

(c) Third order Raman spectrum of 968 and $1117 \mathrm{~cm}^{-1}$ modes is shown as dashed line. 
is apparent that a very good overlap is obtained for the $1584 \mathrm{~cm}^{-1}$ Raman mode. If we go back to the case of $532 \mathrm{~nm}$ excitation, we notice that the frequency shift between the pump and the SNE band is in the order of $3000 \mathrm{~cm}^{-1}$, a shift which is too large for any known Raman mode frequency in DOO-PPV. However, we obtain again good spectral overlap if we consider higher order Raman scattering, as shown in Fig. 7. As we can see, the $640 \mathrm{~nm}$ sharp emission peak, on top of the SNE band, overlaps with the second order of the $1584 \mathrm{~cm}^{-1}$ Raman mode (Fig.4), whereas the $629 \mathrm{~nm}$ peak overlaps with the third order of the $968 \mathrm{~cm}^{-1}$ mode. We conclude therefore, that strong Raman scattering gain is observed only when a spectral coincidence is realized between the fundamental, or higher-order Raman mode, and the spectral region of maximum optical gain. We thus argue that the observed Raman gain is not due to a simple stimulated Raman process".

Another interesting characteristic of the DOO-PPV emission under high excitation intensity is the excitation intensity dependence for both the SNE band and Raman gain peaks. The threshold excitation intensity for the Raman gain is almost one order of magnitude higher than that for the SNE ${ }^{12}$. Moreover, the Raman scattering gain seems to saturate at higher excitation intensities than the SNE, and thus dominates the emission spectrum at the highest excitation intensities ${ }^{12}$.

\section{CONCLUSIONS}

We have investigated DOO-PPV emission spectra at high excitation intensities of order $2+50 \mathrm{MW} / \mathrm{cm}^{2}$ and discovered very narrow $(0.3-1.0 \mathrm{~nm})$ emission lines superimposed on a relatively broad SNE band of $\sim 10 \mathrm{~nm}$ width. The appearance of these spectral features can be explained by Raman scattering gain of the first, second and third order Raman active modes of the polymer, the strongest of which is the ring mode at $1584 \mathrm{~cm}^{-1}$. Raman gain appears at relatively high excitation intensities whenever the polymer Raman active lines of the first, or higher orders coincide with the exciton SNE band.

\section{ACKNOWLEDGMENTS}

We would like to thank S.V. Frolov for the preparation of polymer films.

This work was supported in part by the DOE, grant No. FG-03-95 ER 45490, and by the New Energy and Industrial Development Organization (NEDO) 1997 program in Japan.

\section{REFERENCES}

1. ICSM Proceedings, Synth. Metals 84, pp. 455-476, 1997

2. N. Tessler, G.J. Denton \& R.H. Friend, "Lasing from conjugated-polymer microcavities", Nature, 382, pp. 695-697, 1996.

3. F. Hide, M.A. Dias-Garcia, B.J. Schwartz, M.R. Andersson, Q. Pei and A. Heeger, "Semiconducting polymers: a new class of solid-state laser materials", Science, 273, pp. 1833-1836, 1996.

4. S.V. Frolov, W. Gellermann, M. Ozaki, K. Yoshino and Z.V. Vardeny, "Cooperative emission in $\pi$ conjugated polymer thin films", Phys. Rev. Lett., 78, pp. 729-732, 1994.

5 H.J. Brouwer, V.V. Krasnikov, A. Hilberer and G. Hadziioannou, "Blue superradiance from neat semicon ducting alternating copolymer films", Adv. Mater., 8, pp. 935-937, 1996.

6 S.V. Frolov, M. Ozaki, W. Gellermann, Z.V. Vardeny and K. Yoshino, , "Mirrorless lasing in conducting polymer poly(2,5-dioctyloxy-p-phenylenevinylene) films", Jpn. J. Appl. Phys., 35, p. L1371, 1996.

7. S.V. Frolov, M. Shkunov, Z.V. Vardeny, M. Ozaki and K. Yoshino, "Laser action in conducting polymers", Proceedings SPIE Vol. 3145, 1997

8. A. Yariv, Quantum Electronics, John Wiley and Sons, Inc., New York, 1967.

9. F.T. Arecchi, E.O. Schulz-Dubois, Laser handbook, volume 2, North-Holland Publishing Company, Amster dam, 1972.

10. A. Sakamoto, Y. Furukawa, M. Tasumi, "Infrared and Raman studies of poly(p-phenylenevinylene) and its model compounds", J. Phys. Chem., 96, pp. 1490-1494, 1992.

11. N. Bloembergen, Encounters in Nonlinear Optics, World Scientific, Singapore, p. 339, 1996

12. M.N. Shkunov, W. Gellermann, Z.V. Vardeny and K. Yoshino (to be published). 